\title{
Remarks on the fission barriers of super-heavy nuclei
}

\author{
S. Hofmann ${ }^{1,2}$, S. Heinz ${ }^{1}$, R. Mann ${ }^{1}$, J. Maurer ${ }^{1}$, G. Münzenberg ${ }^{1}$, S. Antalic ${ }^{3}$, W. Barth ${ }^{1}$,
}

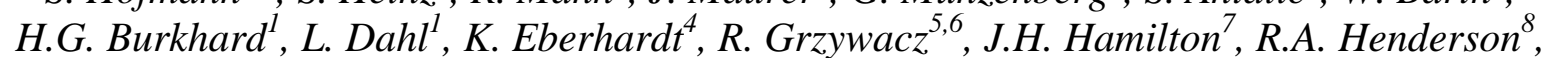
J.M. Kenneally ${ }^{8}$, B. Kindler ${ }^{1}$, I. Kojouharov ${ }^{1}$, R. Lang ${ }^{1}$, B. Lommel ${ }^{1}$, K. Miernik ${ }^{5,9}$, D. Miller ${ }^{6}$, K.J. Moody ${ }^{8}$, K. Morita $^{10}$, K. Nishio ${ }^{11}$, A.G. Popeko ${ }^{12}$, J.B. Roberto ${ }^{5}$, J. Runke ${ }^{4}$, K.P. Rykaczewski ${ }^{5}$, C. Scheidenberger ${ }^{1}$, D.A. Shaughnessy ${ }^{8}$, M.A. Stoyer ${ }^{8}$, P. Thörle-Pospiech ${ }^{4}$, K. Tinschert $^{1}$, N. Trautmann ${ }^{4}$, J. Uusitalo ${ }^{13}$, and A.V. Yeremin ${ }^{12}$

${ }^{1}$ GSI, Darmstadt, Germany; ${ }^{2}$ Goethe-University, Frankfurt, Germany; ${ }^{3}$ Comenius University, Bratislava, Slovakia; ${ }^{4}$ Johannes Gutenberg-University, Mainz, Germany; ${ }^{5}$ ORNL, Oak Ridge, TN, USA; ${ }^{6}$ University of Tennessee, Knoxville, TN, USA; ${ }^{7}$ Vanderbilt University, Nashville, TN, USA; ${ }^{8}$ LLNL, Livermore, CA, USA; ${ }^{9}$ University of Warsaw, Warsaw, Poland; ${ }^{10}$ RIKEN, Wako, Saitama, Japan; ${ }^{11}$ JAEA, Tokai, Ibaraki, Japan; ${ }^{12}$ JINR, Dubna, Russia; ${ }^{13}$ University of Jyväskylä, Jyväskylä, Finland

Shell-correction energies determine the stability and the fission barriers of Super-Heavy Nuclei (SHN), the latter being a main factor responsible for their production yield. Although recent experiments performed at FLNR in Dubna (see review articles [1,2]) have confirmed the existence of an island of SHN, the site and strength of highest stability is still uncertain. Predicted $\mathrm{Q}_{\alpha}$ values as shown in Fig. la reveal the ambiguity. The macroscopic-micro-scopic (MM) models $[3,4]$ predict a closed proton shell at $\mathrm{Z}=$ 114 and thus increasing $Q_{\alpha}$ values beyond. The chiral mean-field model (CMF) [5] and the semi-empirical model (SE) [6] predict subshells or shells at 120 and 126, respectively, resulting in less steep or even decreasing $\mathrm{Q}_{\alpha}$ values. Experimental data, also shown in Fig. 1a and known up to $Z=116$ do not give preference to a specific model. However, decisive information could be obtained from the $\alpha$-decay properties of elements 118 and 120 . The following discussion supports our search experiment for element 120. The study also reveals an important uncertainty related to the prediction of cross-sections of the synthesis of SHN.

\section{Shell-correction energies}

Model dependent experimental shell-correction energies can be deduced from measured nuclear masses by subtraction of theoretically determined liquid-drop masses. In our case, however, absolute experimental nuclear masses are not known, but relative values can be determined for nuclei within an $\alpha$-decay chain using the experimental $\mathrm{Q}_{\alpha}$ values. Normalizing these relative masses to the theoretical ones at the end of the decay chain, which is closer to the region of known masses, where relatively good agreement was established [7], results in a reliable approximation of masses up to the heaviest nuclei of the $\alpha$-decay chain. In one case, where a relatively long decay chain starting at ${ }^{291} \mathrm{Lv}$ and ending at ${ }^{267} \mathrm{Rf}$ is known, we have performed such an estimate of shell-correction energies. Interestingly, this is the decay chain which would be populated in a $3 \mathrm{n}$ channel of the reaction ${ }^{54} \mathrm{Cr}+{ }^{248} \mathrm{Cm}$.

In Figs. $1 \mathrm{~b}$ and 1c, we have plotted the so determined shell-correction energies and compare them with theoretical values taken from [3] and [8], respectively. The relati- ve masses of the four lightest nuclei from ${ }^{267} \mathrm{Rf}$ to ${ }^{279} \mathrm{Ds}$ were normalized by a least squares fit to the theoretical masses. In order to distinguish between the so determined masses, shell-correction energies and fission barriers from the theoretical ones, we denote those 'experimental data' in the following.

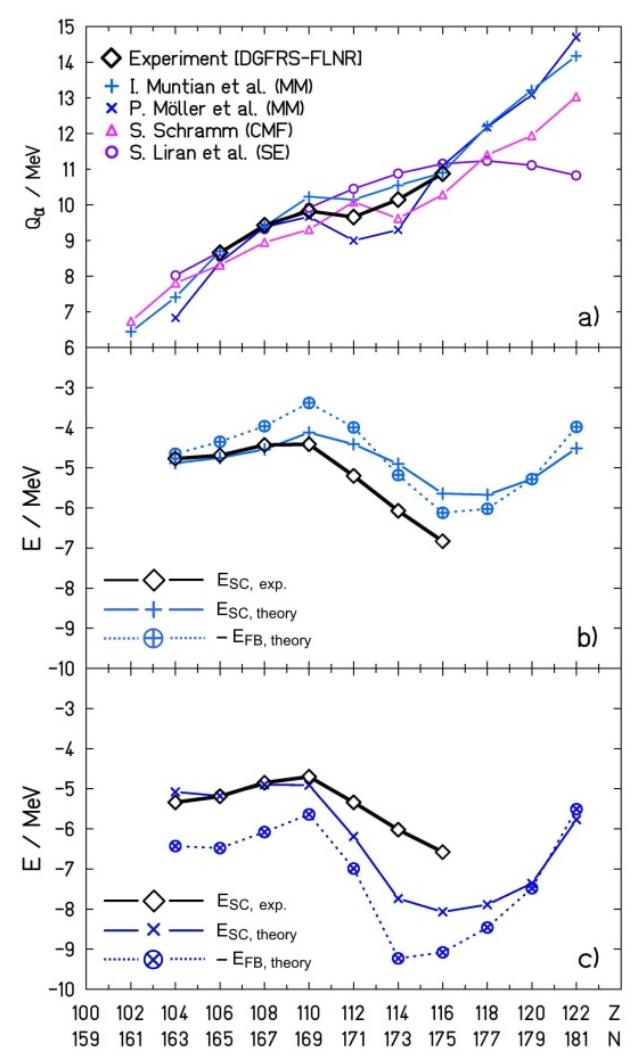

Figure 1: Comparison of experimental and theoretical $\mathrm{Q}_{\alpha}$ values (a) and of experimental and theoretical shellcorrection energies taken from [3] in (b) and from [8] in (c) for nuclei of the $\alpha$-decay chain passing through the isotope ${ }^{291} \mathrm{Lv}$. Also shown in Figs. $1 \mathrm{~b}$ and $1 \mathrm{c}$ are the negative values (for easier comparison with the shellcorrection energies) of the fission barriers connected by dotted lines taken from [9] and [10], respectively. 
The two curves of theoretical shell-correction energies shown in Figs. $1 \mathrm{~b}$ and $1 \mathrm{c}$ and the experimental ones reveal some common features. A moderate increase occurs from ${ }^{267} \mathrm{Rf}$ to ${ }^{279} \mathrm{Ds}$ followed by a sharp decrease (increasing binding energy), up to ${ }^{291} \mathrm{Lv}$. This behaviour is well understood in terms of the shell model having a closed proton shell at $Z=114$ and a low level density above, up to $Z=126$, which shifts the minimum of the shell-correction energies to $Z=116-118$ (see also Fig. 53 in [4]).

The minimum of shell-correction energies is less pronounced in the calculations of [3] than in [8]. The difference at ${ }^{291} \mathrm{Lv}$ is $2.43 \mathrm{MeV}$. However, the shapes and also the absolute values of the two model dependent experimental shell-correction energies obtained from fitting the masses to the lowest four theoretical masses are very similar, both having lowest values of -6.8 and $-6.6 \mathrm{MeV}$, respectively, at ${ }^{191} \mathrm{Lv}$. Especially here we see the importance of the next $\left({ }^{295} 118\right.$ and $\left.{ }^{299} 120\right)$ data points. The $\mathrm{Q}_{\alpha}$ values and deduced shell-correction energies of these two nuclei will provide us with the information of a possible shell or subshell closure at $Z=120$.

In order to rule out energy shifts due to uncertain $Q_{\alpha}$ values of odd-A nuclei, we used the measured $\mathrm{Q}_{\alpha}$ values of neighbouring even-even isotopes with decay chains through ${ }^{290} \mathrm{Lv}$ and ${ }^{292} \mathrm{Lv}$ (see Fig. 4 in [11] and Fig. 11 in [12]) for a similar comparison as shown in Fig. 1. In these cases the $\alpha$ decay occurs with high probability between the ground-states resulting in correct $\mathrm{Q}_{\alpha}$ values. Although the chains of these nuclei end by spontaneous fission of copernicium isotopes, the slope of shell-correction energies between copernicium and ${ }^{294} 118$, respectively, for ${ }^{292} \mathrm{Lv}$ is less than resulting from the calculations [8], but similar as given by the experimental data shown in Fig. 1c for odd isotopes. The trend of shell-correction energies in the case of the odd-A decay chain through ${ }^{293} \mathrm{Lv}$ is similar. This chain is known down to ${ }^{277} \mathrm{Hs}$. We conclude that the shell-correction energies of nuclei of the elements 114 and 116 and with neutron numbers from 174 to 177 decrease less than predicted by the calculations of [8]. The observation of the same trend in four neighbouring decay chains reveals that less strong shell-correction energies could be adopted for the region of isotopes of elements at and beyond 114 for which strong shell-correction energies are predicted in [8].

The shell-correction energy is the main component which determines the fission barrier of SHN. The mutual dependence becomes obvious in a comparison of the shell-correction energy with the inverse of the height of the fission barrier. On this condition we compare in Figs. $1 \mathrm{~b}$ and $1 \mathrm{c}$ the experimental shell-correction energies with the actually calculated fission barriers given in [9] and [10]. The data in [9] were estimated as the mean value of the neighbouring even-even isotopes. In both cases specialization energy is not considered. Whereas in Fig. 1b [9] the curve of the fission barriers is similar to the one of the shell-correction energies, it is significantly lower in the case of Fig. 1c [10] with maximal differences of 1.35 and $1.49 \mathrm{MeV}$ at ${ }^{267} \mathrm{Rf}$ and ${ }^{287} \mathrm{Fl}$, respectively. Obviously, the difference is due to pronounced positive shellcorrection energies at the saddle point in the latter calculation. Even larger is the difference if compared with the experimental shell-correction energies.

\section{Cross-sections}

The height of the fission barrier is primarily responsible for the survival of the compound nucleus (CN) and is, thus, a significant factor contributing to the cross-section of the fusion-evaporation reaction. However, it is not the barrier in the ground-state that is the most important, but the fission barrier height at the excitation energies of the fused system during the evaporation process which is decisive. In the region of SHN, the reduction of fission barriers at excitation energies of several tens of $\mathrm{MeV}$ is determined by the damping of shell-correction energies for deformations from zero up to the saddle point, see e.g. [13].

In the case of hot fusion reactions based on actinide targets, the calculated cross-section depends sensitively from the height of the fission barrier. A change by a factor of 200 was obtained for the reaction ${ }^{48} \mathrm{Ca}+{ }^{249} \mathrm{Cf}$ when the fission barrier of $\approx 5.5 \mathrm{MeV}$ [3] was changed by $1 \mathrm{MeV}$ [14]. Also studied was the influence of the fission barrier on the ER cross-section in $[15,16]$. The $3 n$ crosssection of the reaction ${ }^{48} \mathrm{Ca}+{ }^{238} \mathrm{U}$ was calculated for fission barriers of $4.5,5.5$ and $6.5 \mathrm{MeV}$ resulting in crosssections of $0.23,5.0$ and $30 \mathrm{pb}$, respectively. Considering the uncertainties of fission barriers suggested by a comparison of Figs. 1b and 1c, we have to conclude that calculated cross-sections will differ by one or two orders of magnitude using different model predictions of fission barriers and their dependence on the excitation energy if the entrance channel effects are kept unchanged.

In the case that the relatively high fission barriers shown in Fig. 1c are used in the calculations of crosssections, then the resulting low $\mathrm{CN}$ fission probability is compensated by a strong probability for re-separation of projectile and target nuclei in the entrance channel. The balance becomes especially important for synthesis of nuclei in the region from ${ }^{279} \mathrm{Ds}$ to ${ }^{287} \mathrm{Fl}$, where the theoretical fission barriers of [10] increase rapidly. If, however, the increase of fission barriers is more moderate as calculated in [9] or suggested by the experimental shellcorrection energies, then, the probability for re-separation with increasing $Z_{1} Z_{2}$ has to be more moderate, too. From these arguments follow the conclusions that predicted cross-sections for elements 118 and 120, which use the fission barriers of [10] and which reproduce well the cross-sections of elements 114 and 116, could be higher due to a smaller probability for re-separation in the entrance channel.

\section{References}

[1] Yu.Ts. Oganessian, JPG: NPP 34, R165 (2007).

[2] J.H. Hamilton et al., ARNPS 63, 383 (2013).

[3] I. Muntian et al., Acta Phys. Pol. B 34, 2073 (2003).

[4] P. Möller et al., ADND Tables 66, 131 (1997). 
[5] S. Schramm, Phys. Rev. C 66, 064310 (2002).

[6] S. Liran et al., Phys. Rev. C 62, 047301 (2000).

[7] E. Min. Ramirez et al., Science 337, 1207 (2012).

[8] P. Möller et al., ADND Tables 59, 185 (1995).

[9] M. Kowal et al., Phys. Rev. C 82, 014303 (2010).

[10] P. Möller et al., Phys. Rev. C 79, 064304 (2009).

[11] S. Hofmann, Russian Chem. Rev. 78, 1123 (2009).

[12] S. Hofmann, Lect. Notes Phys. 764, 203 (2009).

[13] J.C. Pei et al., Phys. Rev. Lett. 102, 192501 (2009).

[14] K. Siwek-Wilczyńska et al., IJMP E 19, 500 (2010).

[15] M.G. Itkis et al., Phys. Rev. C 65, 044602 (2002).

[16] V.I. Zagrebaev et al., Phys. At. Nucl. 66, 1033 (2003). 